\title{
Biaxial strain tuning of interlayer excitons in bilayer $\mathrm{MoS}_{2}$
}

\author{
Felix Carrascoso ${ }^{1}$, Der-Yuh Lin ${ }^{2}$, Riccardo Frisenda ${ }^{1, *}$, Andres Castellanos-Gomez ${ }^{1, *}$ \\ ${ }^{1}$ Materials Science Factory, Instituto de Ciencia de Materiales de Madrid (ICMM), Consejo Superior de \\ Investigaciones Científicas (CSIC), Sor Juana Inés de la Cruz 3, 28049 Madrid, Spain. \\ ${ }^{2}$ National Changhua University of Education, Bao-Shan Campus, No. 2, Shi-Da Rd, Changhua City 500, \\ Taiwan, R.O.C \\ * riccardo.frisenda@csic.es, andres.castellanos@csic.es
}

\begin{abstract}
We show how the excitonic features of biaxial $\mathrm{MoS}_{2}$ flakes are very sensitive to biaxial strain. We find a lower bound for the gauge factors of the A exciton and B exciton of ($41 \pm 2) \mathrm{meV} / \%$ and $(-45 \pm 2) \mathrm{meV} / \%$ respectively, which are larger than those found for single-layer $\mathrm{MoS}_{2}$. Interestingly, the interlayer exciton feature also shifts upon biaxial strain but with a gauge factor that is systematically larger than that found for the A exciton, $(-48 \pm 4) \mathrm{meV} / \%$. We attribute this larger gauge factor for the interlayer exciton to the strain tunable van der Waals interaction due to the Poisson effect (the interlayer distance changes upon biaxial strain).
\end{abstract}


The isolation of atomically thin $\mathrm{MoS}_{2}$ by mechanical exfoliation in 2010 opened the door to study the intriguing optical properties of this $2 \mathrm{D}$ semiconductor material. ${ }^{1-3}$ In fact, $\mathrm{MoS}_{2}$ and other members of the transition metal dichalcogenide family show a rich plethora of exitonic physical phenomena, present even at room temperature. Mak et al. and Splendiani et al. observed a strong thickness dependent photoluminescence emission and a direct-to-indirect band gap transition. ${ }^{1,2} \mathrm{MoS}_{2}$ also has tightly bound negative trions, an exciton quasiparticle composed of two electrons and a hole, ${ }^{4,5}$ and several groups in parallel reported the valley polarization, the selective population of one valley, by pumping with circularly polarized light. ${ }^{6-8}$ Moreover, heterostructures built with these 2D systems have also attracted the interest of the scientific community because of the presence of interlayer excitons: excitons formed by electrons and holes that live in different layers. ${ }^{9-13}$ Very recently, Gerber et al. and Slobodeniuk et al. demonstrated that naturally stacked bilayer $\mathrm{MoS}_{2}(2 \mathrm{H}$ - polytype) also presents interlayers excitons, with high binding energy, that can be observed at room temperature ${ }^{14,15}$ and Niehues et al. demonstrated that uniaxial strain could be used to tune the energy of the interlayer exciton. ${ }^{16}$

In this work we employ biaxial strain to modify the band structure, and thus the excitonic resonances, in bilayer $\mathrm{MoS}_{2}$ flakes. We observe that both the $\mathrm{A}$ and $\mathrm{B}$ excitons, as well as the interlayer exciton, substantially redshift upon biaxial tension. Interestingly, unlike to what has been reported for uniaxial strain, we found that the interlayer exciton is more effectively tuned upon straining than the A and B excitons. We attribute this effect to a modification of the interlayer interaction as an in-plane biaxial expansion of the bilayer $\mathrm{MoS}_{2}$ is expected to come hand-by-hand of an out-of-plane compression due to the $\mathrm{MoS}_{2}$ Poisson's ratio. 
$\mathrm{MoS}_{2}$ flakes were prepared by mechanical exfoliation of bulk natural molybdenite (Moly Hill mine, QC, Canada) with Nitto tape (SPV 224). The cleaved $\mathrm{MoS}_{2}$ flakes are then transferred to a Gel-Film (WF 4x 6.0 mil Gel-Film from Gel-Pak®, Hayward, CA, USA). The flakes are optically identified, and their number of layers are determined from quantitative analysis of transmission mode optical microscopy images and microtransmittance/reflectance spectroscopy. ${ }^{17-19}$ Once a suitable bilayer $\mathrm{MoS}_{2}$ flake is located it is transferred onto a polypropylene (PP) substrate by an all-dry deterministic transfer method..$^{20,21}$

(a)

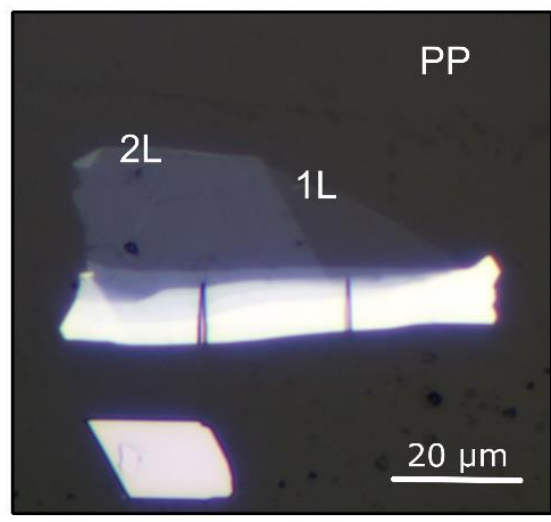

(b)

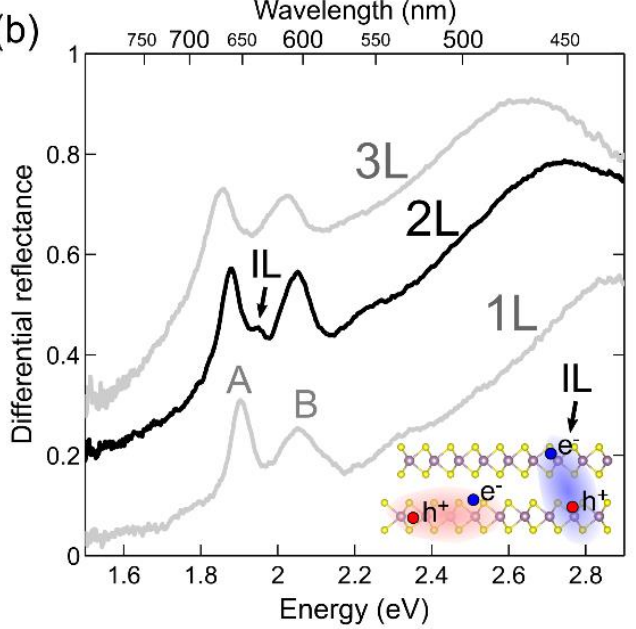

Figure 1. (a) Reflection mode optical microscopy image of a $\mathrm{MoS}_{2}$ flake with single- and bi-layer regions (highlighted with $1 \mathrm{~L}$ and $2 \mathrm{~L}$ respectively) transferred onto the surface of a polypropylene (PP) substrate. (b) Differential reflectance spectra acquired on single-, bi- and tri-layer $\mathrm{MoS}_{2}$ flakes on PP. The A and B excitons are clearly visible for all these thicknesses while the interlayer (IL) exciton, present in multilayer $\mathrm{MoS}_{2}$, is more clearly visible for the bilayer flake.

Figure 1(a) shows a reflection mode optical microscopy image of a $\mathrm{MoS}_{2}$ flake transferred onto PP. Figure 1(b) shows differential reflectance spectra acquired on a mono-, bi- and tri-layer $\mathrm{MoS}_{2}$ flake with a home-built micro-reflectance microscope. We address the reader to Ref. ${ }^{22}$ for technical details about the experimental setup. To obtain the differential reflectance spectra we first collect the light reflected from the substrate $\left(R_{\mathrm{s}}\right)$ 
by means of a fiber-coupled compact CCD spectrometer (see Materials and Methods). Then we collect the light reflected by the desired $\operatorname{MoS}_{2}$ flake $\left(R_{\mathrm{f}}\right)$ and we calculate the differential reflectance as: $\Delta R / R=1-R_{\mathrm{f}} / R_{\mathrm{s}} \cdot{ }^{19,23}$ All the spectra displayed in Figure 1(b) show two strong transitions in all of them assigned to the $\mathrm{A}$ and $\mathrm{B}$ excitons $(\sim 1.9 \mathrm{eV}$ and $\sim 2.05 \mathrm{eV}$ respectively) originated from direct band gap transitions at the $\mathrm{K}$ point of the Brillouin zone. ${ }^{1,2}$ Interestingly, in bilayer $\mathrm{MoS}_{2}$ one can see another prominent peak between the $\mathrm{A}$ and $\mathrm{B}$ excitons. That peak can be also observed in trilayer and even multilayer $\mathrm{MoS}_{2}$ but it cannot be as easily resolved as in the case of bilayer $\mathrm{MoS}_{2}$. This feature in the reflectance spectra have been recently demonstrated (through temperature dependent optical spectroscopy studies, magneto-optical measurements and density functional theory calculations) to be originated by the generation of interlayer (IL) excitons. ${ }^{14,15,24}$ These excitons are, similarly to the A and B excitons, due to direct transitions at the $\mathrm{K}$ point but unlike them the electron and hole are spatially separated in the different $\mathrm{MoS}_{2}$ layers (see the cartoon in Figure 1(b)).

In order to biaxially strain the $\mathrm{MoS}_{2}$ bilayers we exploit the large thermal expansion mismatch between the PP substrate $\left(\sim 130 \times 10^{-6} \mathrm{~K}^{-1}\right)$ and $\mathrm{MoS}_{2}\left(1.9 \times 10^{-6} \mathrm{~K}^{-1}\right)^{25}$. PP has also a relatively high Young's modulus (1.5-2 GPa) for a polymer, which is essential to guarantee an optimal strain transfer from substrate to flake. One can then biaxially stretch (or compress) the flakes by warming up (or cooling down) the substrate. ${ }^{26-28} \mathrm{We}$ used a Peltier element to control the temperature of the substrate around room temperature (27$\left.28^{\circ} \mathrm{C}\right)$ that allows us to cool down to $17^{\circ} \mathrm{C}(-0.13 \%)$ and to warm up to $95^{\circ} \mathrm{C}(+0.87 \%)$. The substrate temperature can be translated to biaxial expansion/compression through the thermal expansion coefficient of PP (see the Supporting Information). 
(a)

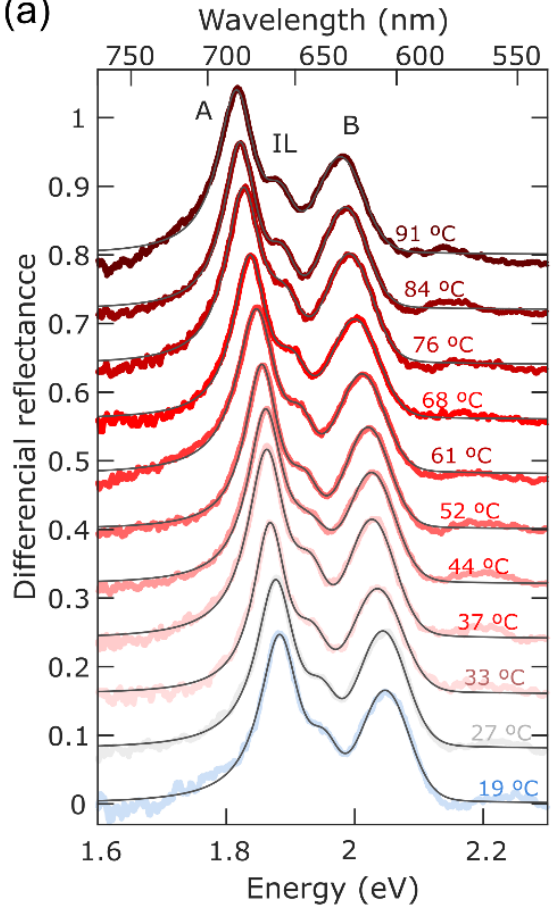

(b)

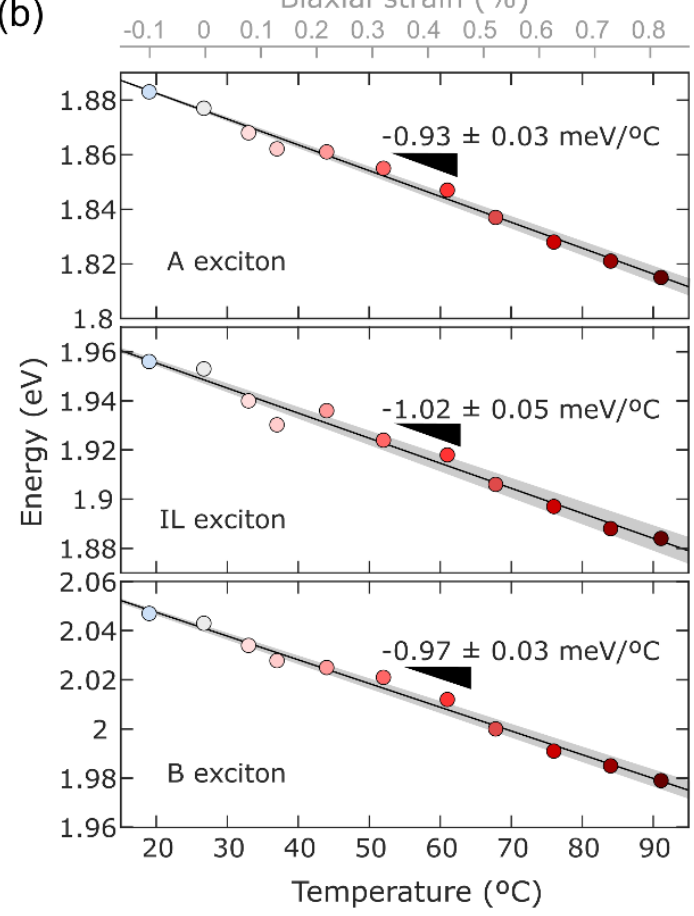

Figure 2. (a) Differential reflectance spectra of a $\mathrm{MoS}_{2}$ bilayer deposited on PP recorded at different temperatures (quadratic polynomial background removed). The black solid lines represent the total fit to the data (composed of three Gaussian peaks). (b) energy of the A, B and IL excitonic peaks extracted from the fit and plotted as a function of the substrate temperature (bottom axis) and of the substrate biaxial strain (top axis).

Figure 2(a) shows the differential reflectance spectra acquired at different substrate temperatures from $19^{\circ} \mathrm{C}$ to $91^{\circ} \mathrm{C}$. The spectra redshift upon temperature increase above room temperature and blueshift when the substrate is cooled down below room temperature. The spectra can be fitted to a sum of Gaussian and Lorentzian peaks in order to extract the energy position of the A, B and IL excitons. The summary of the exciton energy positions is shown in Figure 2(b). From this figure one can extract the spectral shift per ${ }^{\circ} \mathrm{C}$ for the different excitons: $(-0.93 \pm 0.03) \mathrm{meV} /{ }^{\circ} \mathrm{C}$ for the A exciton and $(-0.97$ $\pm 0.03) \mathrm{meV} /{ }^{\circ} \mathrm{C}$ for the $\mathrm{B}$ exciton. In order to disentangle the intrinsic spectral shift expected for $\mathrm{MoS}_{2}$ upon temperature change from that originated from the biaxial strain we fabricated a $\mathrm{MoS}_{2}$ sample on a $\mathrm{Si}$ substrate with $50 \mathrm{~nm}$ of $\mathrm{SiO}_{2}$, which is expected to have a negligible thermal expansion, and we probe the exciton position as a function of 
temperature. We found that for single-, bi- and tri-layer $\mathrm{MoS}_{2}$ all the excitons shift by $0.4 \mathrm{meV} /{ }^{\circ} \mathrm{C}$. By subtracting this intrinsic thermal shift value to the values measured in samples fabricated on PP we can determine the spectral shift induced by biaxial strain. And we can determine the gauge factor, the spectral shift per $\%$ of biaxial strain, by calculating the substrate biaxial expansion (or compression) upon temperature change (see the Supporting Information for details about the thermal expansion calibration of the $\mathrm{PP}$ substrates). The resulting gauge factors for the A and B excitons are $(-41 \pm 2) \mathrm{meV} / \%$ and $(-45 \pm 2) \mathrm{meV} / \%$. Note that these gauge factor values should be considered as a lower bound as we are assuming that all the biaxial expansion of the substrate can be effectively translated to biaxial strain to the $\mathrm{MoS}_{2}$ flake. Due to the Young's modulus mismatch between the PP substrate and the $\mathrm{MoS}_{2}$ the strain transfer efficiency could be lower (and thus we would be underestimating the gauge factor values). ${ }^{26,29}$

It is interesting to note that all the bilayer $\mathrm{MoS}_{2}$ flakes studied here have $\mathrm{A}$ and $\mathrm{B}$ exciton gauge factors that are substantially larger than those found for single-layer flakes which are in the $-(10-25) \mathrm{meV} / \%$ range ${ }^{26}$ (see the Supporting Information for a summary of the measured data in 2 single-layer flakes, other 5 bilayers and one trilayer).

For the interlayer exciton we find a gauge factor of $(-48 \pm 4) \mathrm{meV} / \%$ which is substantially larger than that found for the A exciton. We address the reader to the Supporting Information for datasets acquired on other bilayer $\mathrm{MoS}_{2}$ flakes (with gauge factor up to $-55 \mathrm{meV} / \%$ ) and one trilayer flake that also have larger gauge factor for the interlayer exciton. This contrasts with what has been recently reported for uniaxially strained bilayer $\mathrm{MoS}_{2}$ flakes by Niehues et al. where the gauge factor of the interlayer exciton was slightly lower than that of the A exciton. We attribute the larger gauge factor observed in our experiment to a reduction (or increase) of the bilayer interlayer spacing upon biaxial tension (or compression) as expected from the Poisson effect: as the out-of- 
plane Poisson's ratio of $\mathrm{MoS}_{2}$ is $v_{\mathrm{o}} \sim 0.2$ a biaxial tension of $1 \%$ would yield a reduction of $0.2 \%$ in the interlayer distance. ${ }^{33}$ A similar tunability of the interlayer van der Waals interaction upon biaxial strain has been recently reported in black phosphorus by Huang and co-workers. ${ }^{34}$ The strain tunable interlayer distance could explain the large gauge factor observed for bilayer $\mathrm{MoS}_{2}$ upon biaxial strain as Deilmann and Thygesen demonstrated though density functional theory calculations that the interlayer exciton position strongly depends on the interlayer distance. ${ }^{24}$

In Figure 3 we test the reproducibility of the biaxial strain tuning exploiting the thermal expansion of the substrate. We modulated the temperature of the substrate between $\sim 30^{\circ} \mathrm{C}$ and $\sim 40^{\circ} \mathrm{C}$ (see the registered temperature $v s$. time in the top panel of Figure 3). The color map in the bottom panel shows the time evolution of the differential reflectance spectra and the extracted position of the A, IL and B excitons, extracted from fits similarly to Figure 2(a), are displayed with the black lines. This illustrates the power of this method to tune the van der Waals interlayer interaction and thus the interlayer excitons in biaxial $\mathrm{MoS}_{2}$

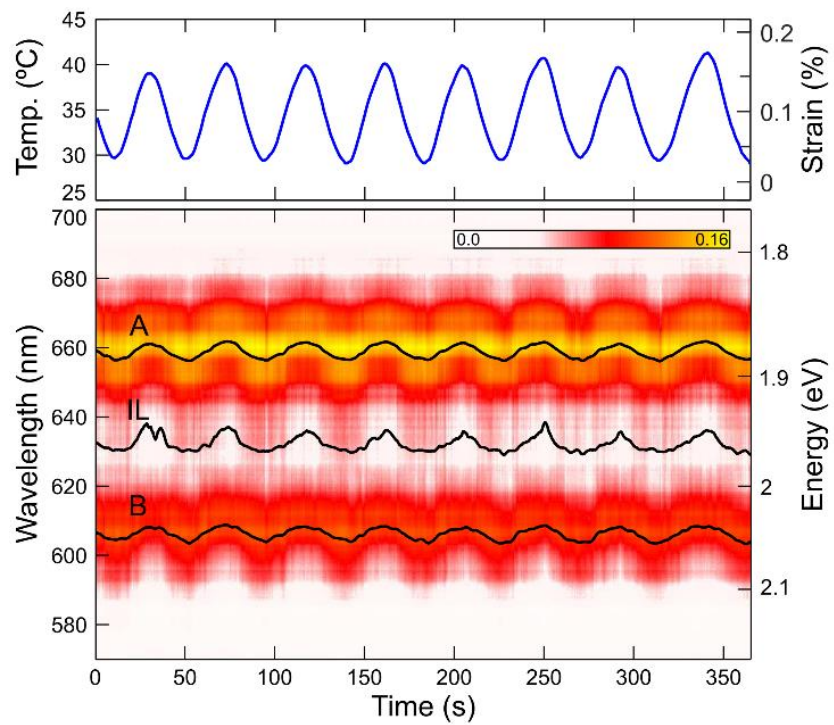

Figure 3. Time evolution of the differential reflectance spectra of bilayer $\mathrm{MoS}_{2}$ (bottom axis) while the temperature of the substrate is cycled between $30^{\circ} \mathrm{C}$ and $40^{\circ} \mathrm{C}$ (top axis). The intensity of the differential reflectance spectra is displayed in the color axis of the colormap. The A, B and IL exciton position is also displayed through the black solid lines. 


\section{CONCLUSIONS}

In summary, we have exploited the large thermal expansion of polypropylene substrates to subject biaxial $\mathrm{MoS}_{2}$ flakes to biaxial strain. We find that the excitons redshift upon biaxial tension with gauge factors that are larger than those reported for monolayer $\mathrm{MoS}_{2}$. Interestingly, the interlayer exciton gauge factor is systematically larger than that of the A and B excitons (contrasting the results reported for uniaxially strained bilayer $\mathrm{MoS}_{2}$ ). We attribute this larger gauge factor of the interlayer exciton to the strain tuning of the van der Waals interaction upon biaxial in-plane straining due to the Poisson effect.

\section{ACKNOWLEDGEMENTS}

This project has received funding from the European Research Council (ERC) under the European Union's Horizon 2020 research and innovation programme (grant agreement $\mathrm{n}^{\circ}$ 755655, ERC-StG 2017 project 2D-TOPSENSE). ACG acknowledge funding from the EU Graphene Flagship funding (Grant Graphene Core 2, 785219). RF acknowledges support from the Spanish Ministry of Economy, Industry and Competitiveness through a Juan de la Cierva-formación fellowship 2017 FJCI-2017-32919. D.-Y.L. acknowledges the financial support from the Ministry of Science and Technology of Taiwan, Republic of China under contract No. MOST 108-2221-E-018-010.

\section{MATERIALS AND METHODS}

Optical microscopy images have been acquired with an AM Scope BA MET310-T upright metallurgical microscope equipped with an AM Scope MU1803 camera with 18 megapixels. The trinocular of the microscope has been modified to connect it to a fiber- 
coupled Thorlabs spectrometer (part number: CCS200/M) to perform the differential reflection spectroscopy measurements. ${ }^{19}$

\section{REFERENCES}

1. Mak, K. F., Lee, C., Hone, J., Shan, J. \& Heinz, T. F. Atomically thin $\mathrm{MoS}_{2}$ : A new direct-gap semiconductor. Phys. Rev. Lett. 105, 136805 (2010).

2. Splendiani, A. et al. Emerging photoluminescence in monolayer $\mathrm{MoS}_{2}$. Nano Lett. 10, 1271-1275 (2010).

3. Castellanos-Gomez, A., Agrat, N. \& Rubio-Bollinger, G. Optical identification of atomically thin dichalcogenide crystals. Appl. Phys. Lett. 96, (2010).

4. Mak, K. F. et al. Tightly bound trions in monolayer MoS2. Nat. Mater. 12, 20711 (2013).

5. Ross, J. S. et al. Electrical control of neutral and charged excitons in a monolayer semiconductor. Nat. Commun. 4, 1474 (2013).

6. Mak, K. F., He, K., Shan, J. \& Heinz, T. F. Control of valley polarization in monolayer $\mathrm{MoS}_{2}$ by optical helicity. Nat. Nanotechnol. 7, 494-8 (2012).

7. Zeng, H., Dai, J., Yao, W., Xiao, D. \& Cui, X. Valley polarization in MoS2 monolayers by optical pumping. Nat. Nanotechnol. 7, 490-3 (2012).

8. Cao, T. et al. Valley-selective circular dichroism of monolayer molybdenum disulphide. Nat. Commun. 3, 887 (2012).

9. Ross, J. S. et al. Interlayer exciton optoelectronics in a 2D heterostructure p-n junction. Nano Lett. 17, 638-643 (2017).

10. Rivera, P. et al. Observation of long-lived interlayer excitons in monolayer MoSe 2-WSe 2 heterostructures. Nat. Commun. 6, 6242 (2015).

11. Yu, Y. et al. Equally efficient interlayer exciton relaxation and improved absorption in epitaxial and nonepitaxial MoS2/WS2 heterostructures. Nano Lett. 15, 486-491 (2014).

12. Baranowski, M. et al. Probing the interlayer exciton physics in a MoS2/MoSe2/MoS2 van der Waals heterostructure. Nano Lett. 17, 6360-6365 (2017).

13. Nagler, P. et al. Interlayer exciton dynamics in a dichalcogenide monolayer heterostructure. 2D Mater. 4, 25112 (2017).

14. Gerber, I. C. et al. Interlayer excitons in bilayer MoS 2 with strong oscillator strength up to room temperature. Phys. Rev. B 99, 35443 (2019).

15. Slobodeniuk, A. O. et al. Fine structure of K-excitons in multilayers of transition metal dichalcogenides. 2D Mater. 6, 25026 (2019).

16. Niehues, I., Blob, A., Stiehm, T. \& de Vasconcellos, S. M. Interlayer excitons in bilayer MoS2 under uniaxial tensile strain. Nanoscale (2019).

17. Niu, Y. et al. Thickness-Dependent Differential Reflectance Spectra of Monolayer and Few-Layer MoS2, MoSe2, WS2 and WSe2. Nanomaterials 8, 725 (2018). 
18. Taghavi, N. S. et al. Thickness determination of MoS 2, MoSe 2, WS 2 and WSe 2 on transparent stamps used for deterministic transfer of 2D materials. Nano Res. $1-5$

19. Frisenda, R. et al. Micro-reflectance and transmittance spectroscopy: A versatile and powerful tool to characterize 2D materials. J. Phys. D. Appl. Phys. 50, (2017).

20. Castellanos-Gomez, A. et al. Deterministic transfer of two-dimensional materials by all-dry viscoelastic stamping. 2D Mater. 1, 011002 (2014).

21. Frisenda, R. et al. Recent progress in the assembly of nanodevices and van der Waals heterostructures by deterministic placement of 2D materials. Chem. Soc. Rev. 47, 53-68 (2018).

22. Frisenda, R. et al. Micro-reflectance and transmittance spectroscopy: A versatile and powerful tool to characterize 2D materials. J. Phys. D. Appl. Phys. 50, 074002 (2017).

23. Dhakal, K. P. et al. Confocal absorption spectral imaging of MoS2: Optical transitions depending on the atomic thickness of intrinsic and chemically doped MoS2. Nanoscale 6, 13028-13035 (2014).

24. Deilmann, T. \& Thygesen, K. S. Interlayer excitons with large optical amplitudes in layered van der Waals materials. Nano Lett. 18, 2984-2989 (2018).

25. El-Mahalawy, S. H. \& Evans, B. L. The thermal expansion of 2H-MoS2, 2HMoSe2 and 2H-WSe2 between 20 and 800 C. J. Appl. Crystallogr. 9, 403-406 (1976).

26. Frisenda, R. et al. Biaxial strain tuning of the optical properties of single-layer transition metal dichalcogenides. npj 2D Mater. Appl. 1, 10 (2017).

27. Frisenda, R. et al. Biaxial strain in atomically thin transition metal dichalcogenides. in Proceedings of SPIE - The International Society for Optical Engineering 10353, (2017).

28. Plechinger, G. et al. Control of biaxial strain in single-layer molybdenite using local thermal expansion of the substrate. 2D Mater. 2, (2015).

29. Liu, Z. et al. Strain and structure heterogeneity in MoS 2 atomic layers grown by chemical vapour deposition. Nat. Commun. 5, 5246 (2014).

30. Roldán, R., Castellanos-Gomez, A., Cappelluti, E. \& Guinea, F. Strain engineering in semiconducting two-dimensional crystals. J. Phys. Condens. Matter 27, 313201 (2015).

31. Lloyd, D. et al. Band Gap Engineering with Ultralarge Biaxial Strains in Suspended Monolayer MoS 2. Nano Lett. 16, 5836-5841 (2016).

32. Scalise, E., Houssa, M., Pourtois, G., Afanas'ev, V. \& Stesmans, A. Straininduced semiconductor to metal transition in the two-dimensional honeycomb structure of MoS2. Nano Res. 5, 43-48 (2011).

33. Woo, S., Park, H. C. \& Son, Y.-W. Poisson's ratio in layered two-dimensional crystals. Phys. Rev. B 93, 75420 (2016).

34. Huang, S. et al. Strain-tunable van der Waals interactions in few-layer black phosphorus. Nat. Commun. 10, 2447 (2019). 


\title{
Supporting Information: Biaxial strain tuning of interlayer excitons in bilayer $\mathrm{MoS}_{2}$
}

\author{
Felix Carrascoso ${ }^{1}$, Der-Yuh Lin ${ }^{2}$, Riccardo Frisenda, ${ }^{1, *}$, Andres Castellanos-Gomez, ${ }^{1, *}$ \\ ${ }^{1}$ Materials Science Factory, Instituto de Ciencia de Materiales de Madrid (ICMM), Consejo Superior de \\ Investigaciones Científicas (CSIC), Sor Juana Inés de la Cruz 3, 28049 Madrid, Spain. \\ ${ }^{2}$ National Changhua University of Education, Bao-Shan Campus, No. 2, Shi-Da Rd, Changhua City 500, \\ Taiwan, R.O.C \\ * riccardo.frisenda@csic.es, andres.castellanos@csic.es
}

Section S1 - Polypropylene substrate thermal expansion calibration

Section S2 - Fitting of the differential reflectance spectra

Section S3 - Additional samples

\author{
Single-layer $\mathrm{MoS}_{2}$ samples \\ Bilayer $\mathrm{MoS}_{2}$ samples \\ Trilayer $\mathrm{MoS}_{2}$ \\ Exciton gauge factor statistics for bilayer MoS2
}

Section S4 - Disentangling temperature and strain effects 


\section{Section S1 - Polypropylene substrate thermal expansion calibration}

a)

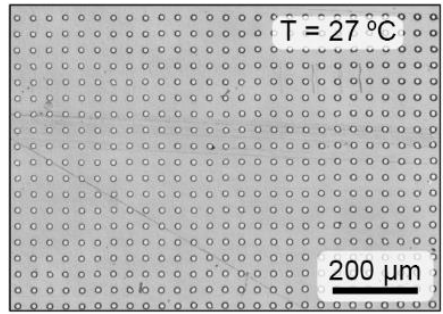

b)

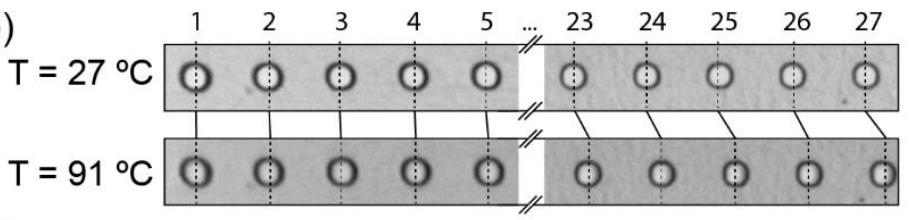

c)

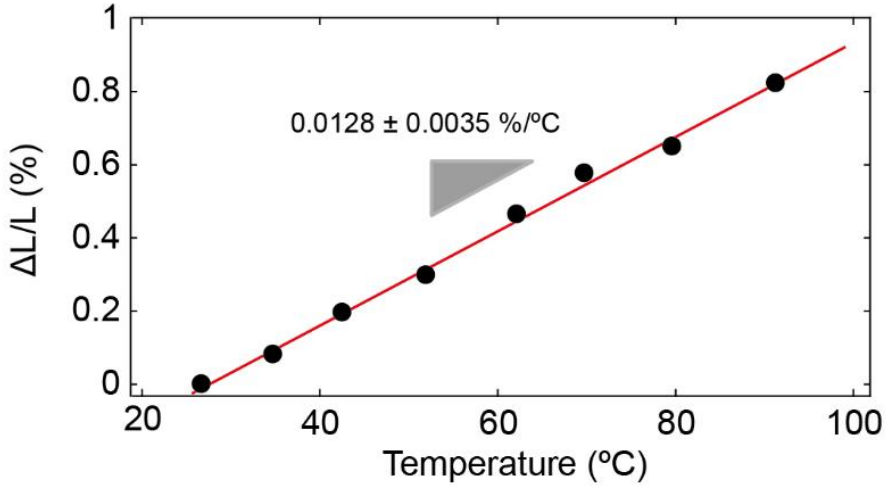

Figure S1. (a) Microscope pictures of a PP substrate with a periodic array of pillars defined by optical lithography at two different temperatures. (b) Zoom in on a row of pillars to show the thermal expansion experienced at high temperature. (c) Relative change $(\Delta \mathrm{L} / \mathrm{L})$ of the spacing between the pillars as a function of temperature. A linear relation can be observed in the experimental temperature range. The thermal expansion coefficient of $\mathrm{PP}$ is determined as $128 \cdot 10^{-6}$ $/ \mathrm{K}$ from the slope of the linear fitting.

\section{Section S2 - Fitting of the differential reflectance spectra}
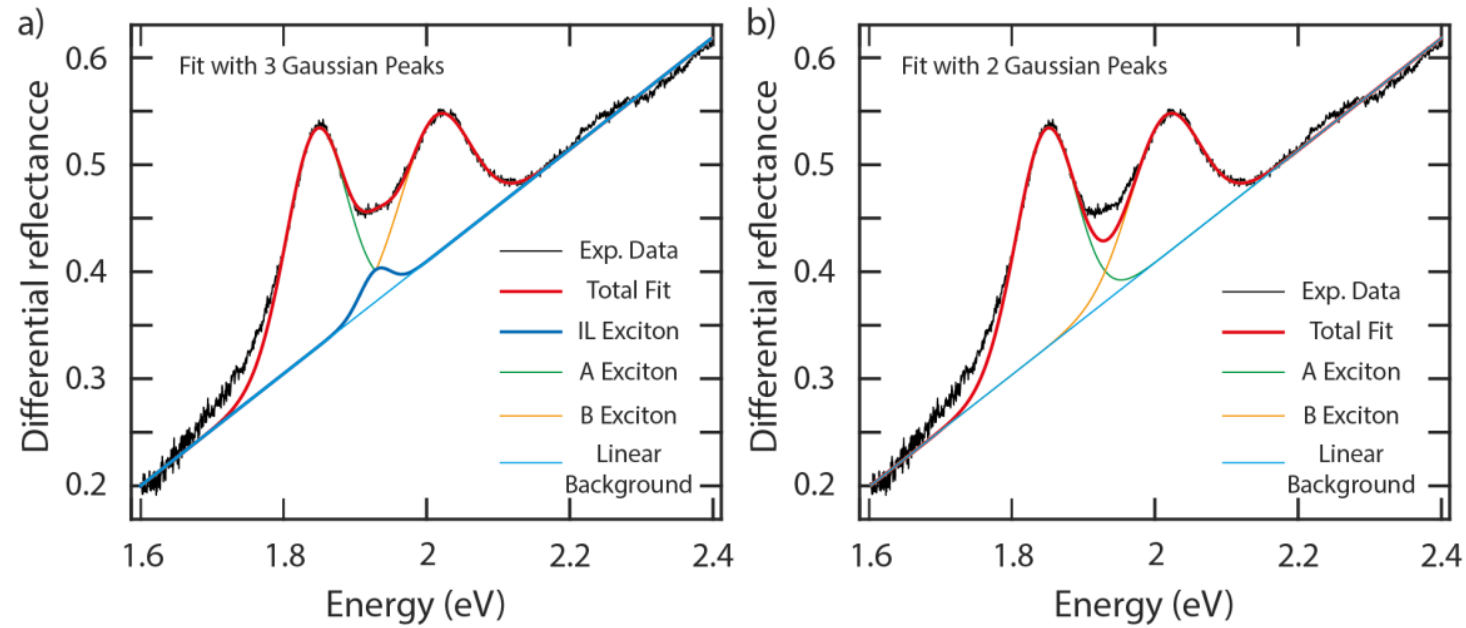

Figure S2. (a) Differential reflectance spectrum of a $\mathrm{MoS}_{2}$ trilayer deposited on PP at $27^{\circ} \mathrm{C}$. The red curve represents the total fit to the data, which is composed by the sum of a linear background and three Gaussian peaks (A, B and IL). (b) Same as (a) with two Gaussian peaks (A and B) instead of three peaks. 


\section{Section S3 - Additional samples}

\section{Single-layer $\mathrm{MoS}_{2}$ samples}
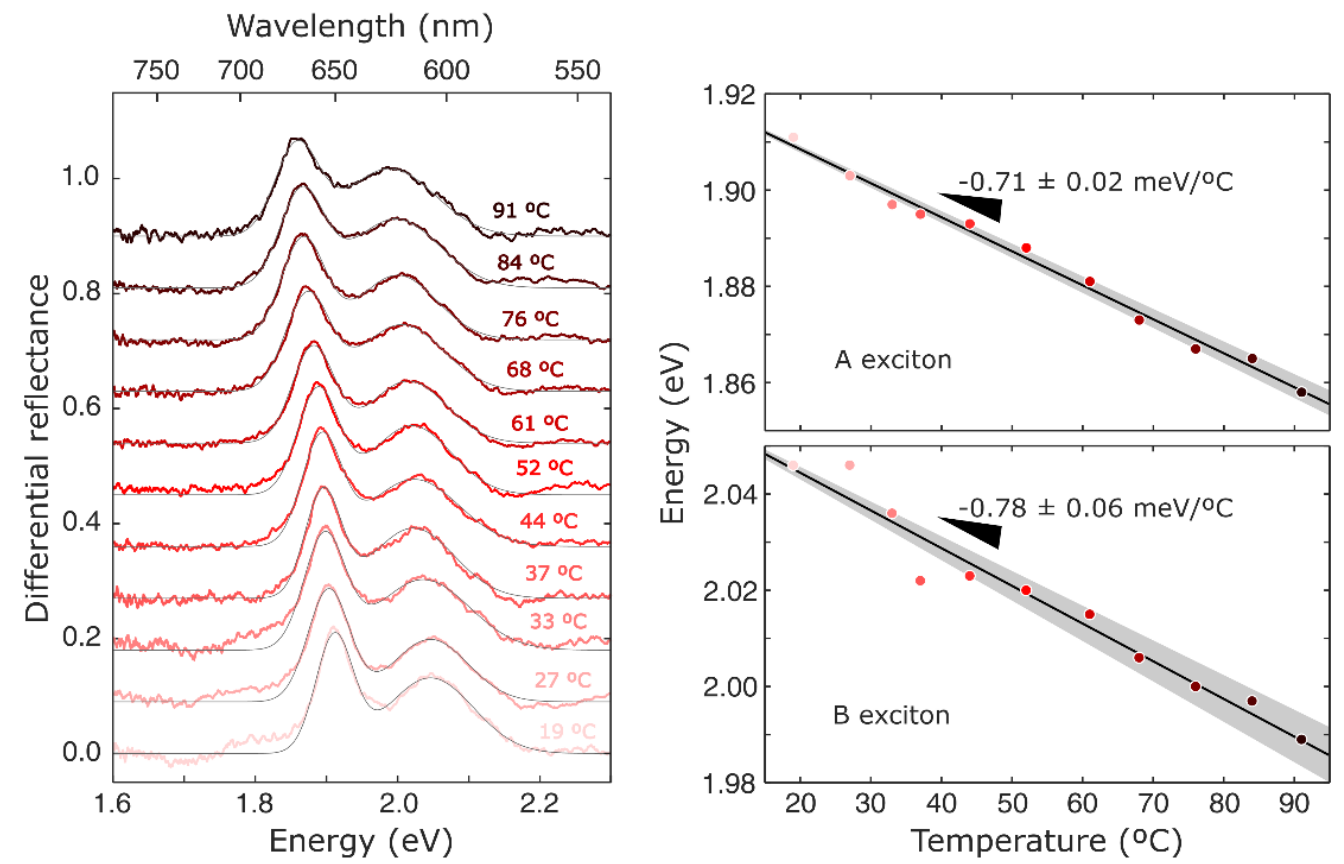

Figure S3. Left: differential reflectance spectra of a $\mathrm{MoS}_{2}$ monolayer deposited on PP recorded at different temperatures. The black solid lines represent the total fit to the data (composed of two Gaussian peaks). Right: energy of the A and B excitonic peaks extracted from the fit plotted as a function of the substrate temperature.
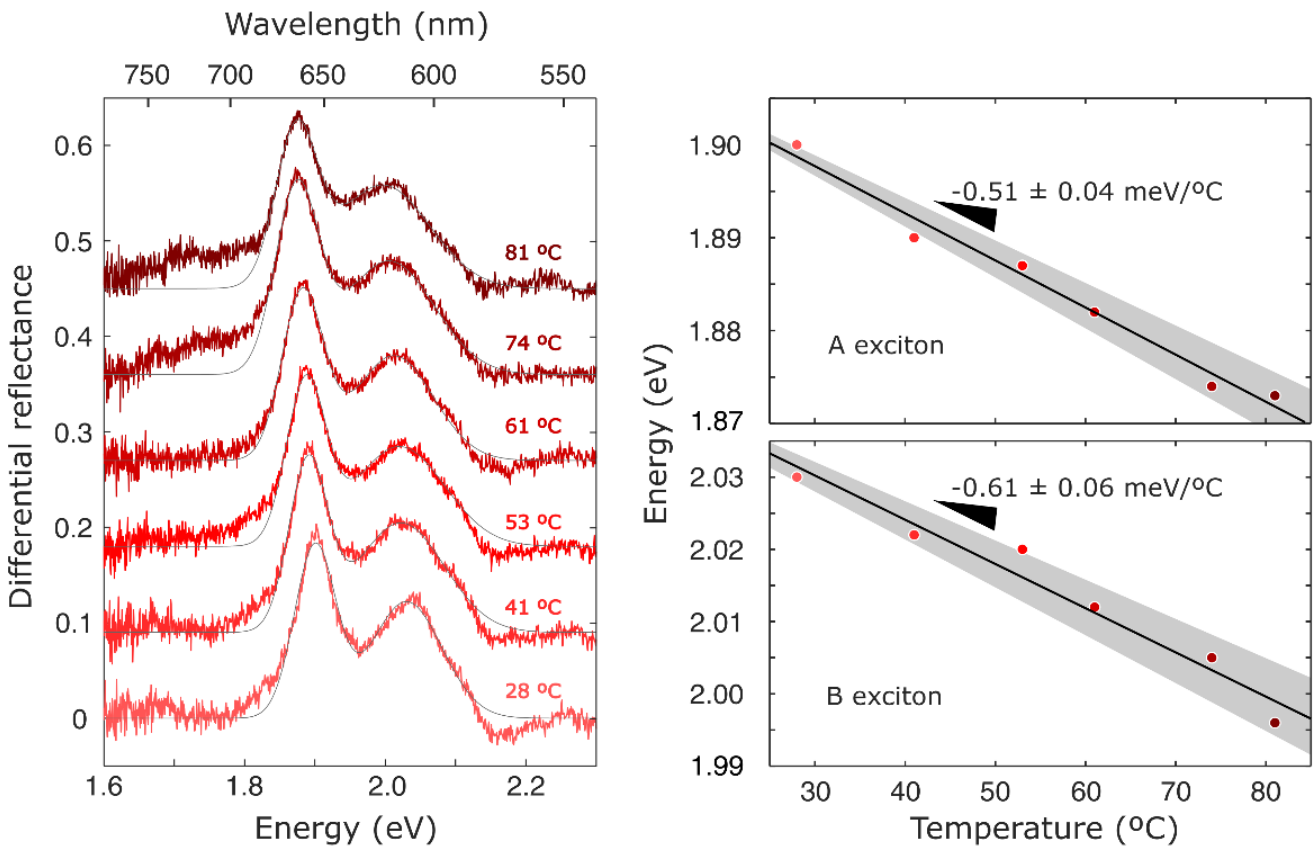

Figure S4. Left: differential reflectance spectra of a $\mathrm{MoS}_{2}$ monolayer deposited on PP recorded at different temperatures. The black solid lines represent the total fit to the data (composed of two Gaussian peaks). Right: energy of the A and B excitonic peaks extracted from the fit plotted as a function of the substrate temperature. 


\section{Bilayer $\mathrm{MoS}_{2}$ samples}
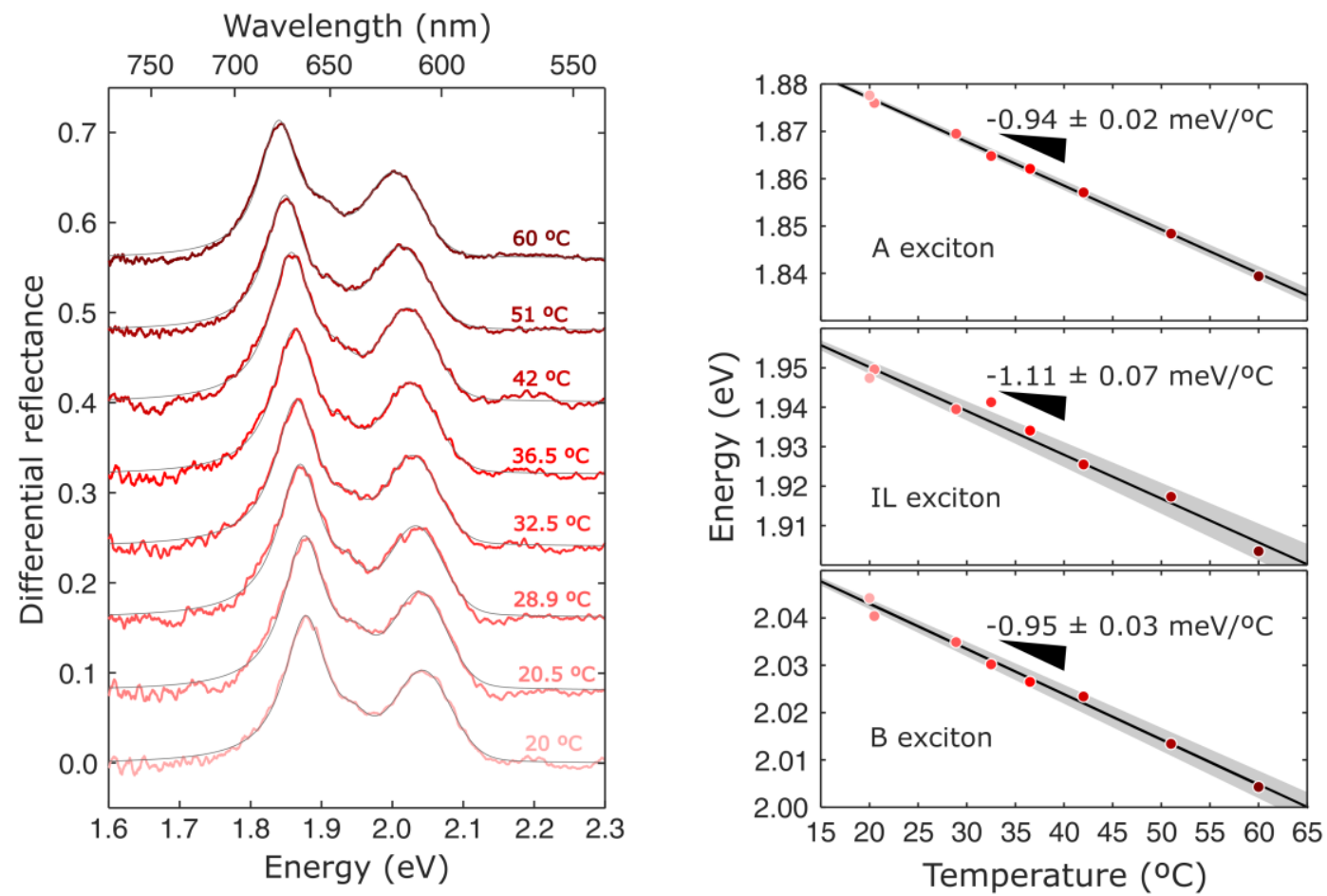

Figure S5. Left: differential reflectance spectra of a $\mathrm{MoS}_{2}$ bilayer deposited on PP recorded at different temperatures. The black solid lines represent the total fit to the data (composed of three Gaussian peaks). Right: energy of the A, B and IL excitonic peaks extracted from the fit plotted as a function of the substrate temperature.
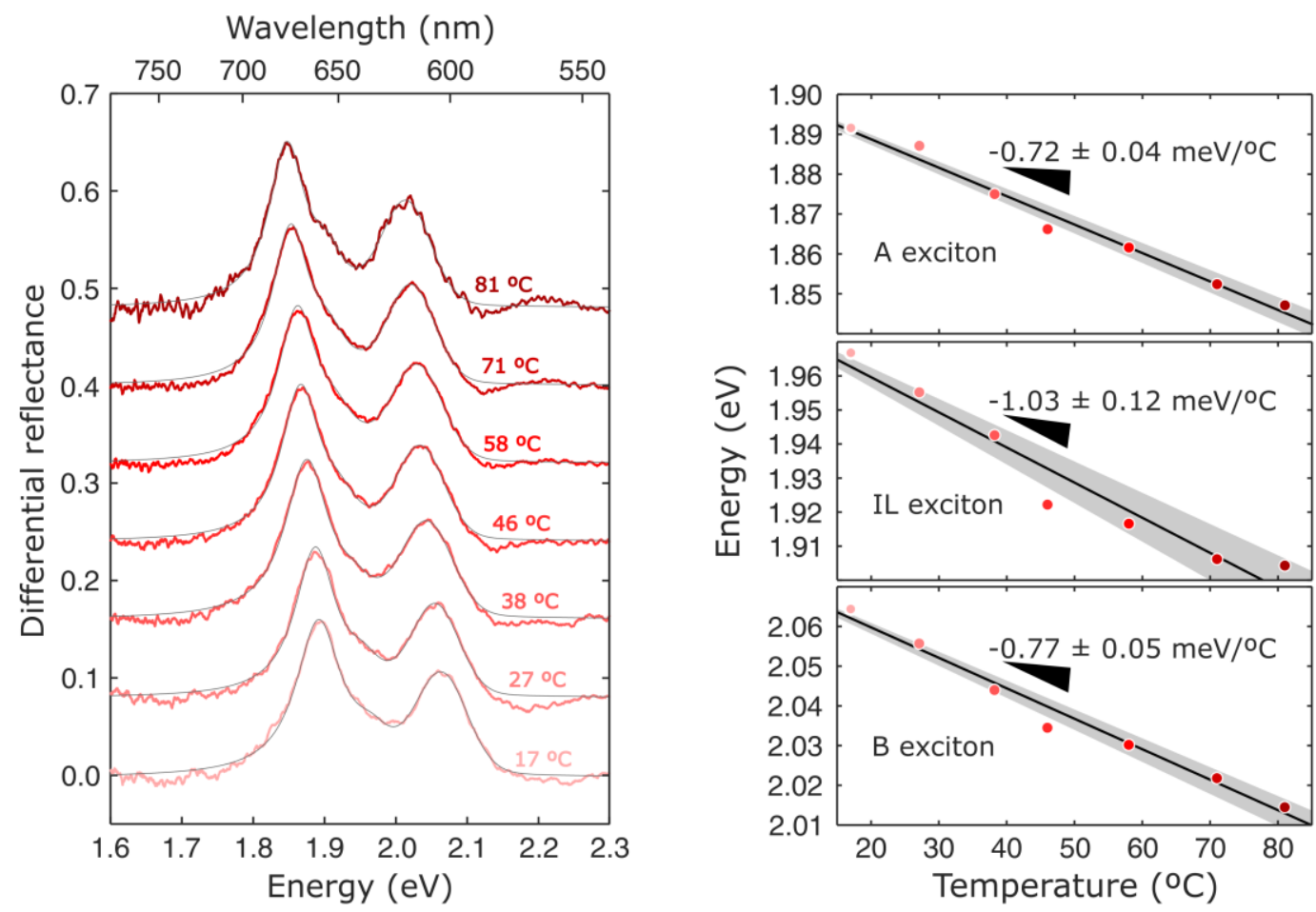

Figure S6. Left: differential reflectance spectra of a $\mathrm{MoS}_{2}$ bilayer deposited on PP recorded at different temperatures. The black solid lines represent the total fit to the data (composed of three Gaussian peaks). Right: energy of the A, B and IL excitonic peaks extracted from the fit plotted as a function of the substrate temperature. 

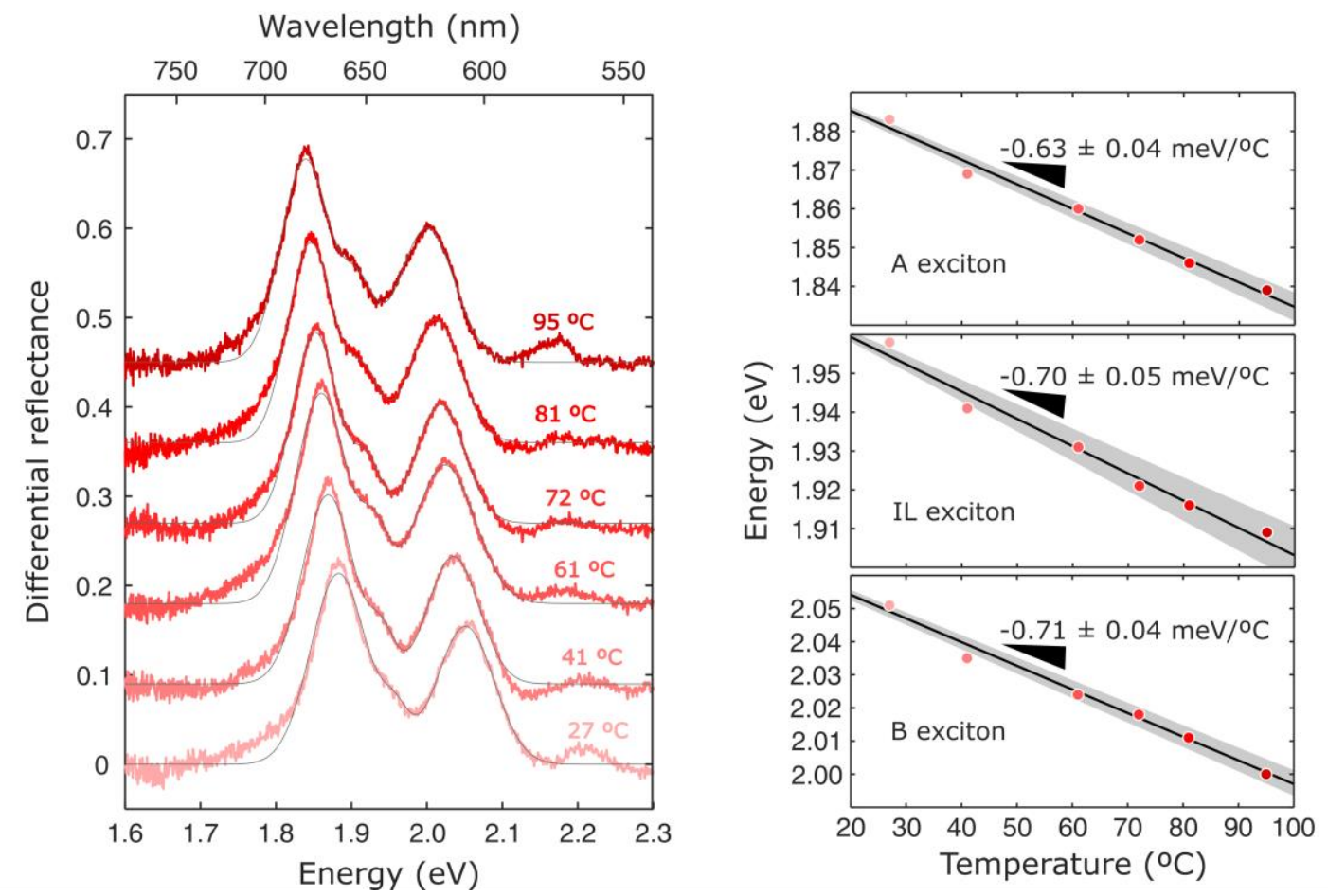

Figure S7. Left: differential reflectance spectra of a $\mathrm{MoS}_{2}$ bilayer deposited on PP recorded at different temperatures. The black solid lines represent the total fit to the data (composed of three Gaussian peaks). Right: energy of the A, B and IL excitonic peaks extracted from the fit plotted as a function of the substrate temperature.
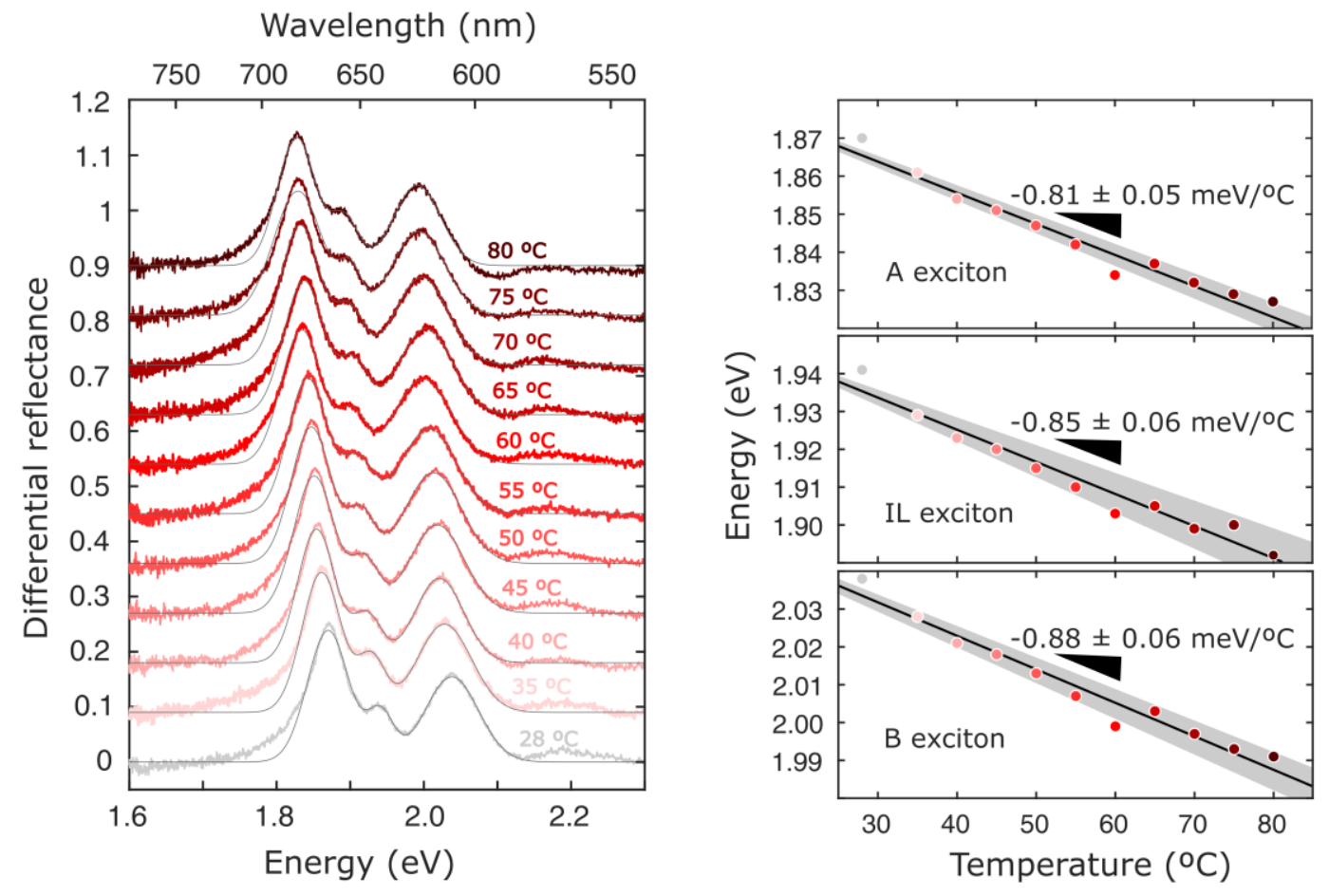

Figure S8. Left: differential reflectance spectra of a $\mathrm{MoS}_{2}$ bilayer deposited on PP recorded at different temperatures. The black solid lines represent the total fit to the data (composed of three Gaussian peaks). Right: energy of the A, B and IL excitonic peaks extracted from the fit plotted as a function of the substrate temperature. 

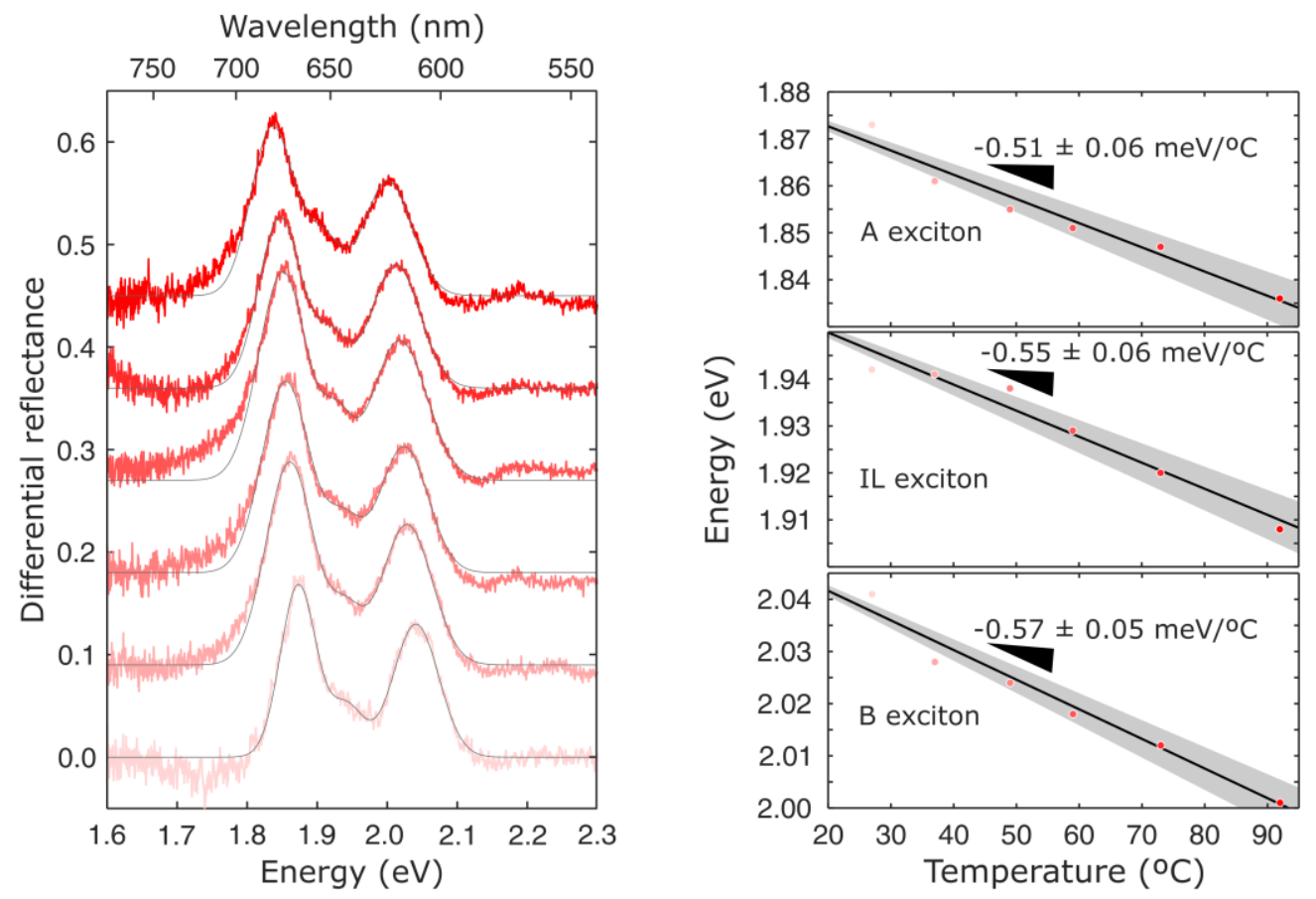

Figure S9. Left: differential reflectance spectra of a $\mathrm{MoS}_{2}$ bilayer deposited on PP recorded at different temperatures. The black solid lines represent the total fit to the data (composed of three Gaussian peaks). Right: energy of the A, B and IL excitonic peaks extracted from the fit plotted as a function of the substrate temperature.

\section{Trilayer MoS2}
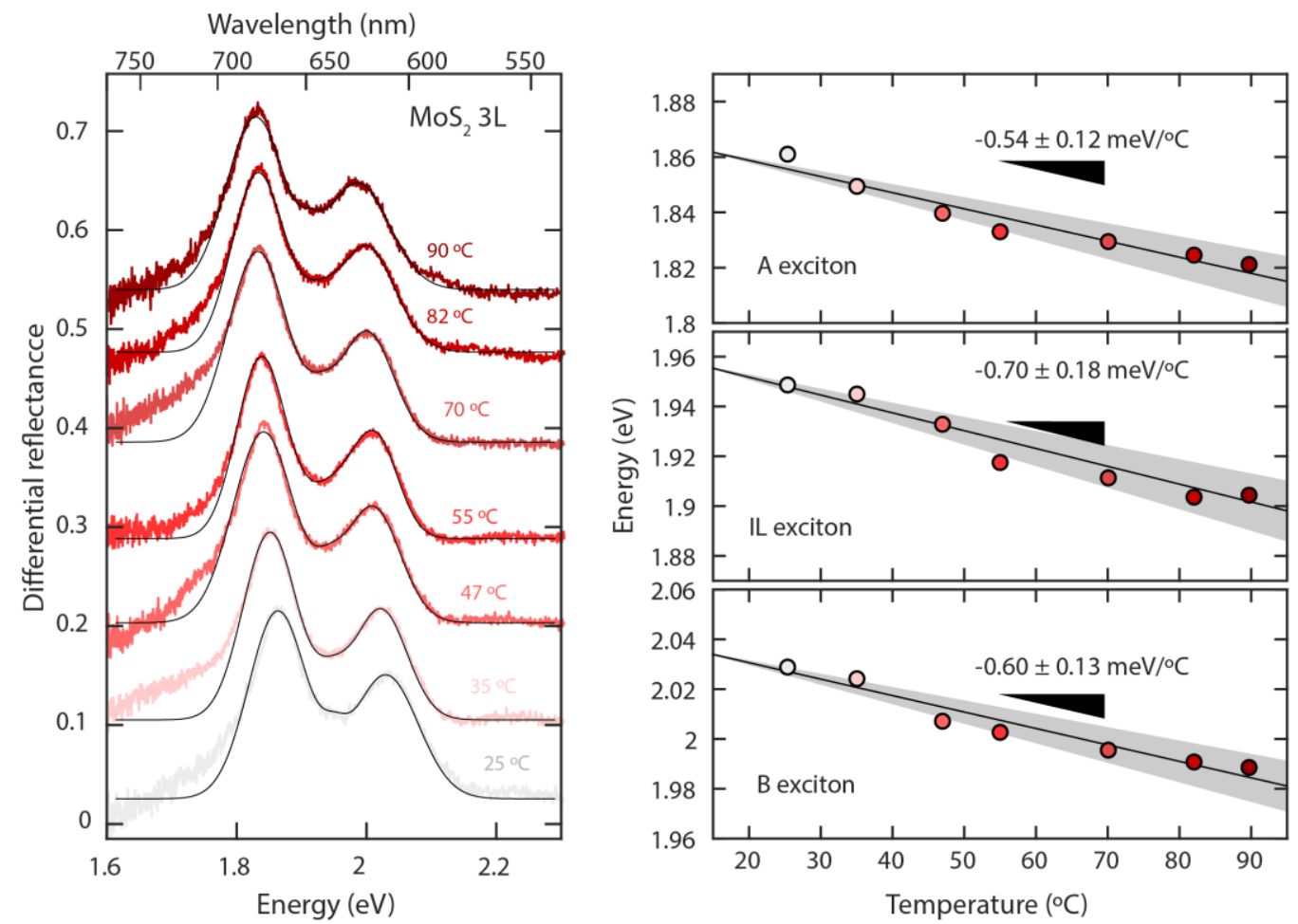

Figure S10. Left: differential reflectance spectra of a $\mathrm{MoS}_{2}$ trilayer deposited on PP recorded at different temperatures. The black solid lines represent the total fit to the data (composed of three Gaussian peaks). Right: energy of the A, B and IL excitonic peaks extracted from the fit plotted as a function of the substrate temperature. 


\section{Exciton gauge factor statistics for bilayer $\mathrm{MoS}_{2}$}

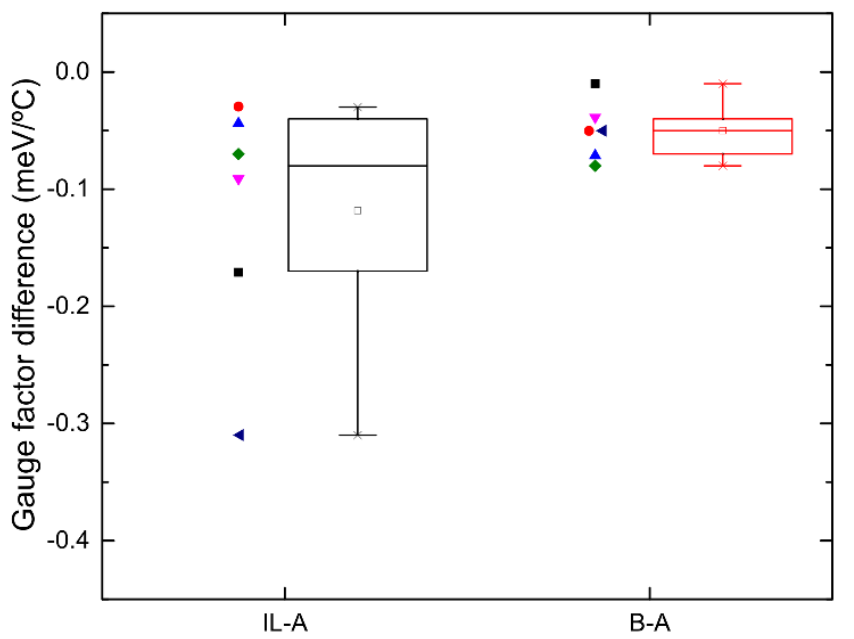

Figure S11. Statistics of the experimental differences between the gauge factors of the B and IL excitons with respect to the A exciton gauge factor. The filled symbols represent the difference between the gauge factors measured for the A, B and IL excitonic peaks in the six bilayer samples studied while the box plots represent the statistical variation. The square points indicate the mean values, the horizontal lines within the boxes the median values, the boxes indicate the quartile values and the vertical lines the maximum and minimum values.

\section{Section S4 - Disentangling temperature and strain effects}

In our experiments the biaxial strain is applied by changing the temperature of the PP substrate therefore we need a way to disentangle the effect arising simply by the temperature change from the effects originated by the biaxial strain. Therefore, we performed a set of measurements on $\mathrm{SiO}_{2} / \mathrm{Si}$, a substrate with a negligible thermal expansion $\sim 1 \cdot 10^{-6} \mathrm{~K}^{-1}$ (as compared with the $128 \cdot 10^{-6} \mathrm{~K}^{-1}$ of the PP substrate). We selected substrates with $50 \mathrm{~nm} \mathrm{SiO}_{2}$ capping layer because they allow to directly resolve the exciton position from differential reflectance measurements. For other $\mathrm{SiO}_{2}$ thicknesses the substrate Fresnel interference could be so strong that hampers the exciton observation. 

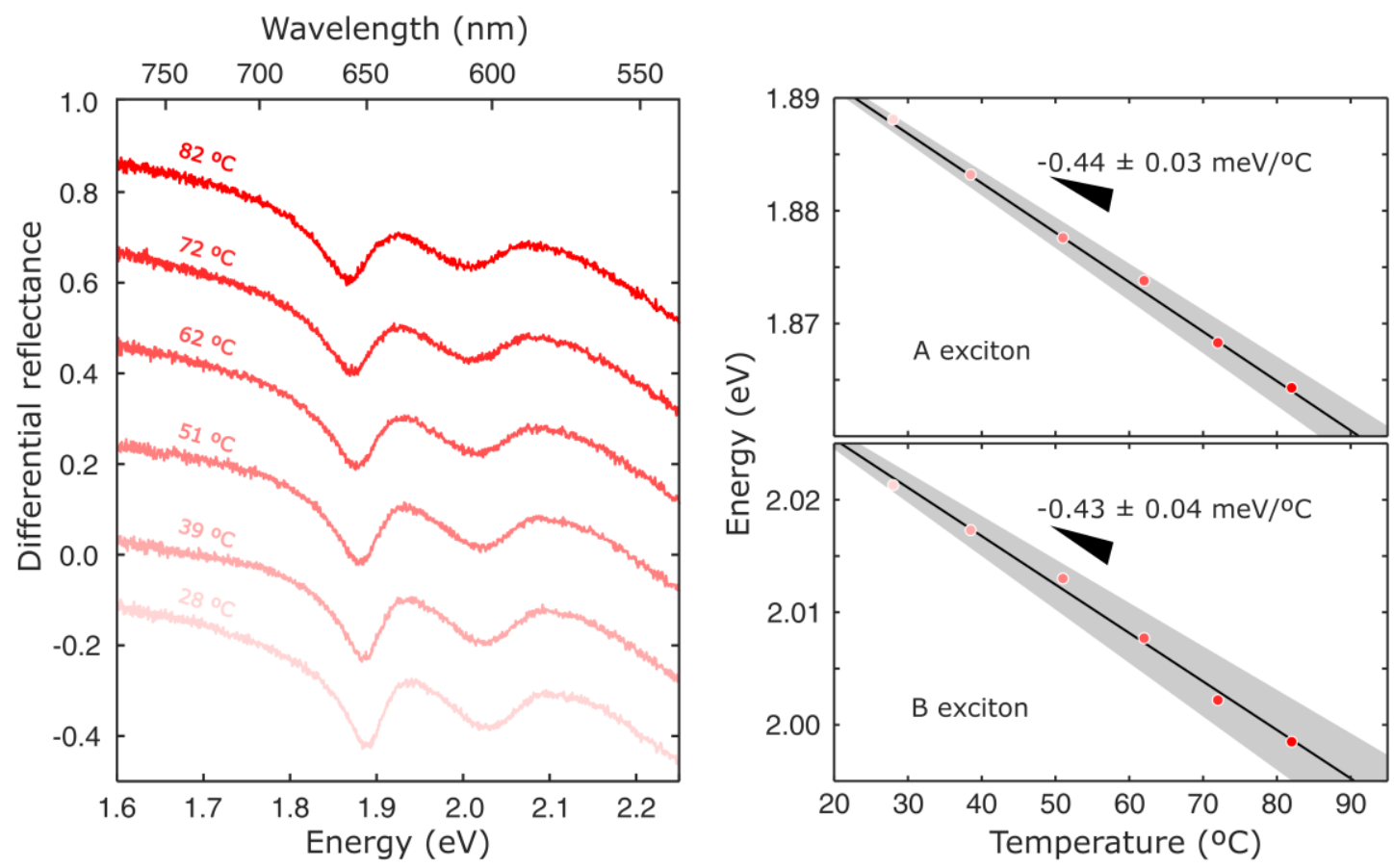

Figure S12. Left: differential reflectance spectra of a $\mathrm{MoS}_{2}$ monolayer deposited on $50 \mathrm{~nm} \mathrm{SiO} / / \mathrm{Si}$ recorded at different temperatures. Right: energy of the $\mathrm{A}$ and $\mathrm{B}$ excitonic peaks plotted as a function of the substrate temperature.
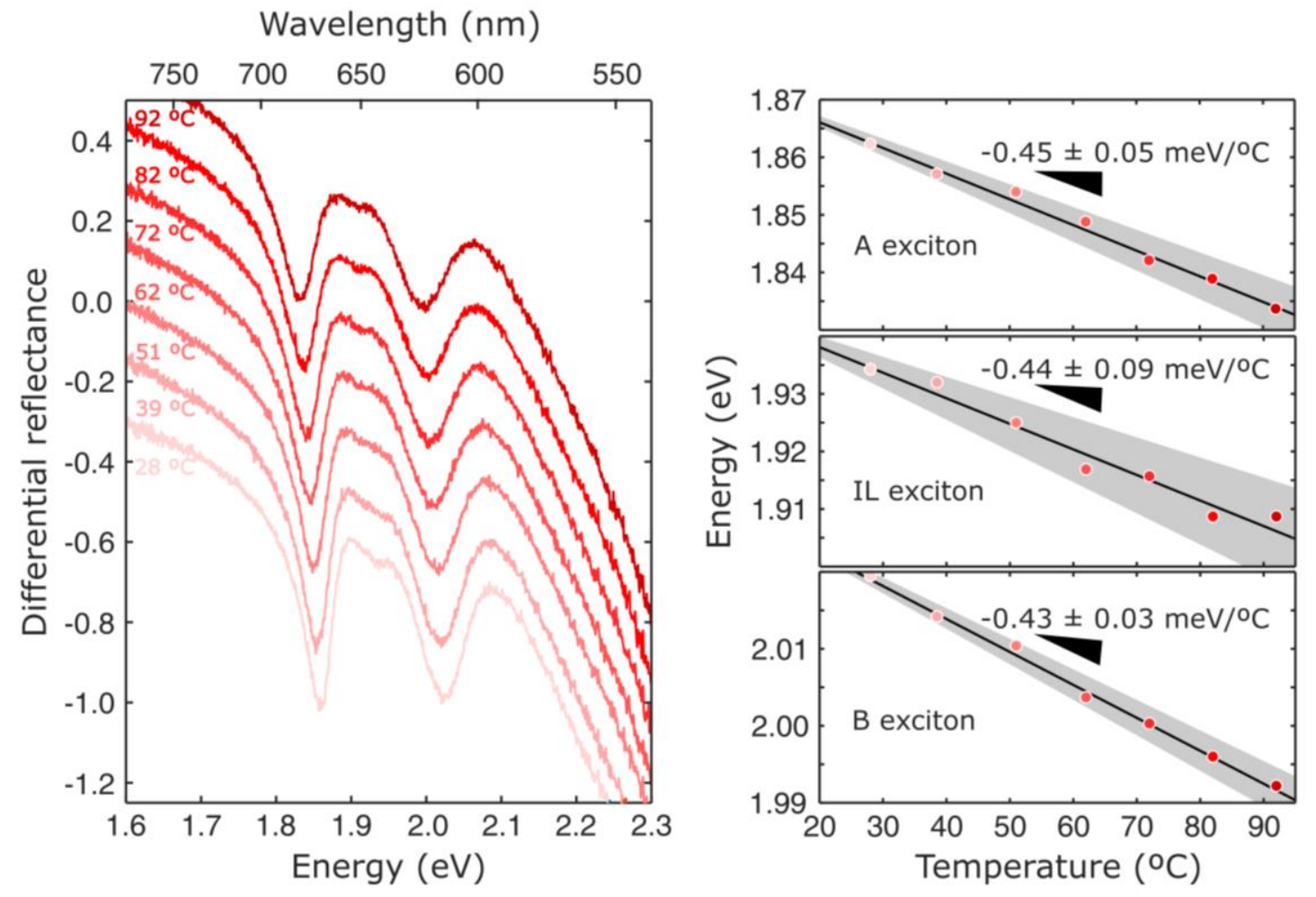

Figure S13. Left: differential reflectance spectra of a $\mathrm{MoS}_{2}$ bilayer deposited on $50 \mathrm{~nm} \mathrm{SiO} / / \mathrm{Si}$ recorded at different temperatures. Right: energy of the A, B and IL excitonic peaks plotted as a function of the substrate temperature. 

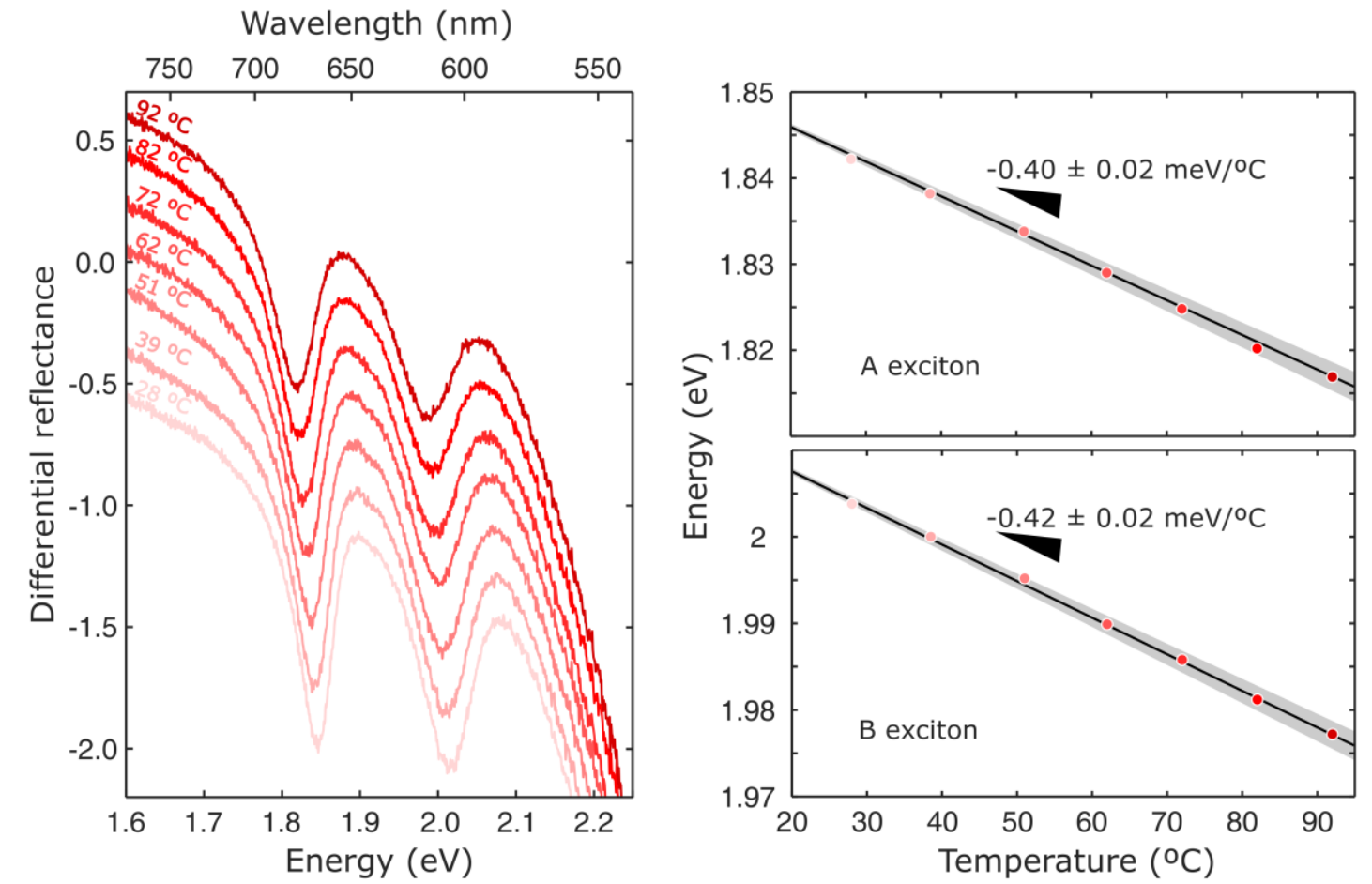

Figure S14. Left: differential reflectance spectra of a $\mathrm{MoS}_{2}$ trilayer deposited on $50 \mathrm{~nm} \mathrm{SiO} / \mathrm{Si}$ recorded at different temperatures. Right: energy of the A and B excitonic peaks plotted as a function of the substrate temperature. 